\title{
83. NUEVAS CITAS PARA LA PROVINCIA DE GRANADA (ANDALUCÍA, ESPAÑA)
}

\author{
Francisco Bruno NAVARRO, Laura BAENA y M. Teresa VIZOSO
}

\begin{abstract}
New cites for Granada province (Spain).
Palabras clave. Guadix-Baza, corología, ecología.

Key words. Guadix-Baza, corology, ecology.
\end{abstract}

Este trabajo constituye el resultado de un estudio de la flora en cultivos agrícolas abandonados de la depresión de Guadix-Baza y de algunas otras campañas de muestreo en lugares poco estudiados desde el punto de vista florístico de la provincia de Granada, en general agrosistemas. Se relacionan por tanto algunos táxones que constituyen nuevas citas provinciales, alguna segunda cita de interés y otras cuya constancia era dudosa, oral o sin testimonio de herbario.

La nomenclatura empleada ha sido la establecida por Flora Ibérica (Castroviejo et al. [eds.], 1986-2001) para las familias publicadas, y en su defecto, la Flora Vascular de Andalucía Occidental (Valdés et al. [eds.], 1987) y Flora Europaea (Tutin et al. [eds.], 1972-1980). Para la comprobación de las citas se ha empleado igualmente Flora Ibérica y las recogidas en las publicaciones de mayor relevancia para la Península Ibérica publicadas por Velayos et al. (1991a, 1991b y 1992) y Velayos \& Castilla (1993), así como revisiones recientes y notas corológicas breves que se especifican en el texto.

Los testimonios de herbario a los que hacemos referencia se depositaron en las instalaciones del Herbario de la Universidad de Granada (GDA).

\section{Alyssum linifolium Wild.}

GRANADA: Guadix. Rambla de Becerra. 30S VG9142. 990 m. F.B. Navarro. 27-03-2001. (GDA
43823). Pastos anuales en sustratos pedregosos; Granada: Entre la Puebla de Don Fadrique y Cañadas de Cañepla. 20-VI-1989. G. Blanca \& M. Cueto. (GDA 45616).

Especie de amplia distribución por toda la franja este de la Península Ibérica que no había sido citada con anterioridad en la provincia de Granada, en los pisos bioclimáticos mesomediterráneo seco y semiárido sobre sustratos básicos.

\section{Loeflingia baetica Lag.}

GRANADA: Guadix. Rambla de Becerra. 30S VG9143. 950 m. F.B. Navarro. 4-06-1998. (GDA 43486). Pastos anuales en sustratos arenosos; Granada: Guadix. Rambla de Becerra. 30S VG9043. 950 m. 25-05-2000. F.B. Navarro. (GDA 43488). Pastos anuales en sustratos arenosos.

Constituye la primera cita para la provincia de Granada siendo la de mayor altitud para la especie en la Península Ibérica puesto que su distribución se restringe a zonas arenosas costeras del sur-suroeste peninsular y Marruecos. En la localidad de estudio es una especie muy escasa que aparece sobre el lecho arenoso de ramblas constituído por materiales silíceos descalcificados en el piso mesomediterráneo semiárido.

Anthyllis vulneraria L. subsp. gandogeri (Sagorski) W. Becker

GRANADA: Guadix. Rambla de Becerra. 30S VG9142. 950 m. 10-06-2000. F.B. Navarro. (GDA 43569). Pastizales subnitrófilos en cultivos abandonados.

Especie de distribución iberonorteafricana muy extendida por la Península Ibérica pero que no había sido citada con anterioridad en la provincia de Granada. 


\section{Fumana scoparia Pomel}

GRANADA: Guadix. Rambla de Becerra. 30S VG9141. 980 m. 24-05-1999, F.B. Navarro. (GDA 43835). Romerales y tomillares; Granada: Guadix. Rambla de Bácor. 30S WG0550. 900 m. 13-051997. F.B. Navarro, H. Tenorio y J. Muñoz. (GDA. 455339). Pinar-coscojal sobre margas; Granada: Guadix. Dehesa de los Cuartos. 30S WG0347. 1030 m. 24-04-1998. F.B. Navarro. (GDA 45538). Romerales y espartales; Granada: Villanueva de las Torres. Puntal de Carboneros. 30S VG8749. $800 \mathrm{~m}$. 28-03-1998. F. B. Navarro. (GDA 45540). Romerales y matorrales fruticosos.

Su distribución en la Península se restringe a áreas muy localizadas de la mitad oriental. Estas citas constituyen su ampliación de areal a la provincia de Granada donde se distribuye por toda la depresión de Guadix-Baza formando parte de romerales donde es abundante y convive con otras dos especies de su género, Fumana thymifolia y Fumana hispidula.

\section{Clypeola eriocarpa Cav.}

GRANADA: Guadix. Rambla de Becerra. 30S VG9142. 1000 m. 10-04-2001. F.B. Navarro. (GDA 43828). Eriales y cultivos abandonados; Granada: Huéscar, Base de la Sa Jureña. 6-VI-1989. C. Morales \& A.T. Romero. (GDA 45550).

Esta cita constituye la ampliación del areal para esta especie en la provincia de Granada, pues en ésta solo se tenía constancia de su presencia en la Puebla de D. Fadrique (norte de la provincia). Su interés radica en que está considerada como en peligro de extinción (VV.AA., 2000). Se encuentra amenazada por fragmentación severa del hábitat así como la declinación de sus poblaciones debido a la reducción del área, extensión y/o calidad del hábitat. Al ser una especie anual sus poblaciones están sujetas a fluctuaciones en el número de localidades, subpoblaciones e individuos. La población de Rambla de Becerra varía atendiendo al año climatológico, pero en el mejor de los casos es escasa y local. Aparece formando parte de pastizales terofíticos puros entre matorrales fruticosos y espartales en el piso mesomediterráneo secosemiárido.

\section{Lepidium latifolium $\mathrm{L}$.}

GRANADA: Granada. Gójar. 800 m. 10-062001. F.B. Navarro. (GDA 45546). Herbazales húmedos de huertos y vegas de regadío.

Se confirma su presencia en la provincia de Granada tal y como fue referenciada por Losa \& Rivas-Goday (1974) para la Hoya de Baza. Hernández-Bermejo \& Clemente in: Castroviejo et ai. [eds.] (1993:326) hacen constancia de la presencia en esta provincia mediante testimonio oral de algún especialista o por referencia bibliográfica fiable, pero no por el material de herbario revisado.

\section{Chenopodium botrys $\mathrm{L}$.}

GRANADA: Guadix. Hernán Valle. $30 \mathrm{~S}$ VG9436. 1160 m. 20-08-1998. F.B. Navarro. (GDA 45545). Barbechos y cultivos abandonados.

Especie rara en el sur peninsular, que no ha sido recogida por Flora Ibérica aunque su presencia en Granada había sido denunciada por FernándezCasas (1972) en la Hoya de Baza y Martínez-Parras et al. (1986) en el Pantano de los Bermejales, confirmando con este testimonio su existencia en nuestra provincia. Aparece de forma muy puntual formando parte de herbazales estivales sobre barbechos en el piso mesomediterráneo seco.

\section{Juniperus thurifera $\mathrm{L}$.}

GRANADA: Alamedilla. El Peñón. 30S VG8261. 850 m. 24-04-1997. F.B. Navarro. (GDA 45544). Parameras sobre sustratos margosos.

Taxon propio de las altas parameras del centro y norte peninsular que aparece de forma relíctica en Andalucía donde do Amaral Franco in: Castroviejo et al. [eds.] (1986:187-188), tan solo lo cita en Jaén y Almería. Sin embargo su presencia ya era conocida en Granada y aparece reflejada en algunos trabajos (Lorite et al., 2000; etc.), pero en ellos no se ratifica su existencia con testimonios de herbario. En Alamedilla permanece la población mejor conservada, aunque existen también ejemplares sueitos en la comarca de Huéscar (cercanías del embalse de San Clemente). Su situación en la comunidad autónoma andaluza merecería algún plan de protección y conservación.

\section{Vella pseudocytisus L. subsp. pseudocytisus}

GRANADA: Orce. Venta Micena. $30 \mathrm{~S}$ WG5579. 20-05-1997. F.B. Navarro, H. Tenorio y J. Muñoz. (GDA 42088); Granada: Puebla de Don Fadrique y Cañada de Cañepla. 22-06-1988. O. Socorro. (GDA 22576). 
Al igual que en el caso anterior en la actualidad es ampliamente conocida la existencia de esta especie en el norte de las provincias de Granada y Almería, aunque Gómez-Campo in Castroviejo et al. [eds.] (1993:415) denota que su presencia en estas provincias es un tanto incierta. Está considerada como una especie en peligro de extinción y viene recogida en el decreto andaluz 104/94 y el Libro Rojo Andaluz (LRA 99-00) de flora amenazada. Sin embargo hasta esta contribución no existían testimonios de herbario puestos a disposición de la comunidad científica.

\section{BIBLIOGRAFÍA}

CASTROVIEJO, S. et al. [eds.] - 19986/2001-Flora Ibérica, vols. 1-8, 14. Real Jardín Botánico de Madrid, C.S.I.C. Madrid.

FERNÁNDEZ-CASAS, J. -1972- Estudio fitogeográfico de la cuenca del Guadiana Menor. Tesis doctoral.

LORITE, J., F.B. NAVARRO, C. SALAZAR \& F. VALLE -2000- Comparative study of Juniperus thurifera L. formations in the Betic mountains (S.E. Spain). ONF-Les dossiers forestiers 6: 53-61.

LOSA, T.M. y S. RIVAS-GODAY -1974- Estudio florístico y geobotánico de la provincia de Almería. Archivos Inst. Aclimatación Almería 13(2): 117-237.

MARTÍNEZ-PARRAS, J.M., M. PEINADO, J. ÁLVAREZ y L. MONJE - 1986- Aplicaciones a la flora de Andalucía oriental. Lazaroa 9: 139145.

TUTIN, T.G. et al. [eds.] -1964/1980- Flora Europaea. Vols. I-V. Cambridge University Press.

VALDÉS, B., S. TALAVERA y F. GALIANO 1987-Flora vascular de Andalucía occidental. 3 vols. Ed. Ketres. Barcelona.

VELAYOS, M. y F. CASTILLA - 1993-Archivos de Flora Ibérica 6(Corología Ibérica, IV). C.S.I.C. Madrid.

VELAYOS, M., F. CASTILLA y R. GAMARRA 1991 a-Archivos de Flora Ibérica 2 (Corología Ibérica, I). C.S.I.C. Madrid.
VELAYOS, M. F. CASTILLA y R. GAMARRA 1991 b- Archivos de Flora Ibérica 3 (Corología Ibérica, II). C.S.I.C. Madrid.

VELAYOS, M. F. CASTILLA y R. GAMARRA 1992- Archivos de Flora Ibérica 5 (Corología Ibérica, III). C.S.I.C. Madrid.

VV. AA. -2000- Lista roja de Flora Vascular Española (valoración según categorías UICN). Conservación vegetal 6 (extra): 11-38.

Aceptado para su publicación en julio de 2002

Dirección de los autores. F.B. NAVARRO: Dpto. Forestal, Centro de Investigación y Formación Agraria (Consejería de Agricultura y Pesca, Junta de Andalucía), Camino de Purchil s/n ${ }^{\circ} 18080$ Granada. E-mail: cifafore@teleline.es; L. BAENA y M.T. VIZOSO: Herbario de la Facultad de Ciencias de la Universidad de Granada, Severo Ochoa s $/ n^{\circ}$ 18001 Granada. E-mail: herbario@ugr.es 